\title{
Suppression of tunicamycin-induced CD44v6 ectodomain shedding and apoptosis is correlated with temporal expression patterns of active ADAM10, MMP-9 and MMP-13 proteins in Caki-2 renal carcinoma cells
}

\author{
YEOUN-HEE KIM and JAE-CHANG JUNG \\ Department of Biology, Kyungpook National University, Daegu 702-701, Republic of Korea
}

Received May 28, 2012; Accepted June 27, 2012

DOI: $10.3892 /$ or.2012.1986

\begin{abstract}
CD44v6 has been shown to coordinate the activation of anti-apoptotic molecules as well as resistance to apoptosis. Here, we investigated CD44v6 ectodomain shedding in Caki-2 human renal carcinoma cells as well as its underlying mechanisms. Exposure of cells to tunicamycin (TM)-induced apoptosis was accompanied by cleavage of caspase-3, PARP-1 and CD44v6 ectodomain. TM-induced apoptosis was also closely associated with endoplasmic reticulum (ER) stress, as shown by increased expression of GRP-78 and CHOP proteins. Furthermore, induction of matrix metalloproteinase (MMP)13, MMP-9 and ADAM10 expression was highly stimulated by tunicamycin in a time- and dose-dependent manner. TM-induced PARP-1 cleavage was significantly inhibited by treatment with GM6001 (a broad spectrum MMP inhibitor), MMP-9/-13 inhibitor and GI254023X (specific ADAM10 inhibitor). In addition, inhibition of all examined MMPs resulted in reversal of TM-induced apoptosis as well as increased cell viability. When considering the functional implications of MMP-9 and ADAM10, it is likely that active MMP-9 and ADAM10 help regulate the cellular levels of CD44v6 through cleavage of CD44v6 ectodomain during TM-induced apoptosis of Caki-2 cells. Collectively, these findings suggest that multiple TM-induced MMPs may cooperate to induce apoptosis.
\end{abstract}

\section{Introduction}

The accumulation of unfolded proteins can be induced by agents such as tunicamycin (TM), a naturally occurring antibiotic that

Correspondence to: Professor Jae-Chang Jung, Developmental Biology Laboratory, Department of Biology, College of Natural Sciences, Kyungpook National University, 1370 Sankyuk-dong, Daegu 702-701, Republic of Korea

E-mail: jcjung@knu.ac.kr

Abbreviations: TM, tunicamycin; MMPs, matrix metalloproteinases; ADAM, a disintegrin and metalloproteinase

Key words: tunicamycin, apoptosis, ADAM10, MMP-9/-13, CD44v6 induces ER stress by inhibiting the first step in the biosynthesis of N-linked oligosaccharides in cells $(1,2)$. Inhibition of N-linked glycosylation by TM results in cell death in various cell types $(1,3,4)$. ER stress triggers a unique pathway of apoptosis that is mediated via activation of ER-resident caspases $(5,6)$.

CD44, a highly glycosylated cell surface adhesion molecule involved in cell-matrix interactions, was originally identified as a receptor for hyaluronate and later found to have affinity for various molecules (7). CD44 has many variant forms, which are generated by alternative splicing of at least 10 exons named v1-v10 (8). Normal cells usually express standard CD44 (CD44s), which lacks the entire group of variant exons $(9,10)$. Soluble CD44v6 is considered as a useful marker of tumor prognosis (11). For example, the malignancy grade of colon cancer is related to the expression of CD44v6 (12). Specifically, tumor progression requires crosstalk between neighboring tumors, wherein the tumor matrix plays an essential role and CD44v6 coordinates tumor matrix-triggered motility and apoptosis resistance (13). Many tumor cells resistant to apoptosis express CD44v6, which co-localizes and interacts with Fas, thereby inhibiting Fas-mediated apoptosis $(13,14)$. Thus, CD44v6 ectodomain shedding may have important effects on the apoptosis of tumor cells.

Matrix metalloproteinases (MMPs) are extracellular zincdependent endopeptidases involved in the degradation and remodeling of the extracellular matrix (ECM) in physiological and pathological processes in vertebrates (15). Since all MMPs are synthesized as inactive proenzymes, their ability to act as proteases is dependent on the presence of appropriate activation mechanisms (16). In addition to their role in ECM turnover, MMPs also target other proteinases, latent growth factors, cell surface receptors, cytokines, cell adhesion molecules and the release of apoptotic Fas ligands (17). Based on structural relationships and substrate specificities, MMPs have been divided into 4 subfamilies: collagenases, stromelysins, gelatinases and membrane-tethered MMPs (MT-MMPs). Further, the ADAM (a disintegrin and metalloproteinase) family of metzincin proteinases is closely related to MMPs (18).

ADAM10 has a wide variety of substrates and can remodel ECM components in addition to membrane proteins (19). ADAM10 has been implicated in the shedding of CD44v6 ectodomain from the cell surface (20-22). Release of CD44v6 
ectodomain from the cell surface results in dynamic regulation of the interaction between CD44 and the ECM. CD44v6 ectodomain cleavage event also initiates the CD44-mediated intracellular signaling pathway (22). The proteolytic activity of MMP-9 (gelatinase B) has been implicated in various physiological and pathological conditions. Specifically, the extracellular domain of CD44 binds MMP-9, which cleaves CD44s as well as CD44v6 $(9,23)$. Further, the MMP-13 (collagenase-3) cascade results in the activation of pro-MMP-9 to MMP-9 (24,25), and the inhibition of MMP-13 prevents MMP-9 activation (25).

Considering the importance of CD44v6 to apoptosis resistance, we determined the expression profiles of MMP-9, MMP-13 and ADAM10, which are responsible for CD44v6 ectodomain shedding during TM-induced apoptosis of Caki-2 cells.

\section{Materials and methods}

Reagents. Caki-2 human renal carcinoma cells were obtained from the American Type Culture Collection (ATCC; Rockville, MD). Tunicamycin (TM) and MTT were purchased from Sigma (St. Louis, MO). GI254023X (ADAM10-specific inhibitor) was obtained from GlaxoSmithKline (Stevenage, UK). MMP-9/-13 inhibitor, GM6001 and anti-MMP-9 were purchased Calbiochem (La Jolla, CA). Monoclonal anti-CD44v6 and polyclonal anti-ADAM10 and MMP-13 antibodies were purchased from Chemicon International, Inc. (Temecula, CA). Monoclonal anti- $\beta$-actin, polyclonal anti-PARP, anti-GADD153, anti-KDEL and anti-procaspase-3 were purchased from Santa Cruz Biotechnology, Inc. (Santa Cruz, CA).

Cell culture. Cells were plated onto 6-well culture plates and maintained at $37^{\circ} \mathrm{C}$ in $5 \% \mathrm{CO}_{2}$ and $95 \%$ air. The culture medium consisted of Dulbecco's modified Eagle's medium (DMEM; Gibco Invitrogen, Carlsbad, CA), 10\% (v/v) fetal bovine serum (FBS) and $1 \%(\mathrm{v} / \mathrm{v})$ penicillin-streptomycin. Cells were plated at a density of $0.6 \times 10^{6}$ cells/well, incubated for $24 \mathrm{~h}$, and then treated with TM $(0.01,0.1$ and $5 \mu \mathrm{g} / \mathrm{ml})$ for $24 \mathrm{~h}$.

Preparation of conditioned medium. After serum cultures for $24 \mathrm{~h}$, cells were washed twice with serum-free DMEM. Then, cells were treated with or without TM $(0.01,0.1$ and $5 \mu \mathrm{g}$ / $\mathrm{ml}$ ) in serum-free DMEM for $24 \mathrm{~h}$, after which serum-free conditioned medium was collected. To examine the levels of MMP-9, MMP-13, ADAM10 and cleaved CD44v6 ectodomain, proteins were precipitated from equal-volume aliquots of supernatant using $10 \%$ ice-cold trichloroacetic acid (TCA). The precipitates were washed twice with $100 \%$ acetone, airdried, dissolved in RIPA buffer and stored at $-20^{\circ} \mathrm{C}$ until use.

Western blot analysis. Equal volumes of conditioned media or equal amounts of protein lysate $(30 \mu \mathrm{g})$ in RIPA buffer were separated by 7.5 or $10 \%$ SDS-PAGE under reducing conditions. After transfer, nitrocellulose membranes were incubated for $1 \mathrm{~h}$ with primary antibodies against anti-CD44v6, ADAM10, MMP-13, PARP, caspase-3, CHOP, GRP78 and $\beta$-actin. The results were visualized using horseradish peroxidase (HRP)-conjugated second antibodies, along with an enhanced chemiluminescence kit.
FACS analysis. Cells $\left(\sim 1 \times 10^{6}\right)$ were suspended in $100 \mu 1$ of PBS, and $200 \mu \mathrm{l}$ of $95 \%$ ethanol were added while vortexing. Then, the cells were incubated at $4^{\circ} \mathrm{C}$ for $1 \mathrm{~h}$, washed with PBS, and resuspended in $250 \mu \mathrm{l}$ of $1.12 \%$ sodium citrate buffer ( $\mathrm{pH}$ 8.4) together with $12.5 \mu \mathrm{g}$ of RNase. Incubation was continued at $37^{\circ} \mathrm{C}$ for $30 \mathrm{~min}$. The cellular DNA was then stained by applying $250 \mu \mathrm{l}$ of propidium iodide $(50 \mu \mathrm{g} / \mathrm{ml})$ for $30 \mathrm{~min}$ at room temperature. The stained cells were analyzed by fluorescent activated cell sorting (FACS) on a FACScan flow cytometer to determine relative DNA content based on red fluorescence.

MTT assay. Cell viability was determined by MTT assay. Caki-2 cells in 96-well plates were incubated with TM $(5 \mu \mathrm{g}$ / $\mathrm{ml}$ ) in the presence of several MMP inhibitors for $24 \mathrm{~h}$. Then, $10 \mu \mathrm{l}$ of stock MTT solution ( $5 \mathrm{mg} / \mathrm{ml}$ in PBS) were added to each well, after which cells were incubated for another $4 \mathrm{~h}$ at $37^{\circ} \mathrm{C}$. The supernatants were then aspirated carefully, and $100 \mu \mathrm{l}$ of DMSO were added to each well. The plates were shaken for an additional $5 \mathrm{~min}$ on a plate shaker, and the absorbance values were read using a Microplate Reader (Bio-Rad, Hercules, CA) at $570 \mathrm{~nm}$.

Zymography. To profile secreted gelatinases, gelatin gel zymography was performed as previously described (15). SDS-containing $8 \%$ polyacrylamide gels were co-polymerized with gelatin as substrates for the identification of MMP-9 in culture medium. Conditioned medium was loaded onto the gel without boiling and electrophoresed under non-reducing conditions. The gels were then shaken for $1 \mathrm{~h}$ in a $2.5 \%$ solution of Triton X-100 at room temperature to remove SDS and were incubated at $37^{\circ} \mathrm{C}$ in reaction buffer $(50 \mathrm{mM}$ Tris- $\mathrm{HCl}, \mathrm{pH} 7.5$; $10 \mathrm{mM} \mathrm{CaCl}_{2}$ ) overnight to allow proteinases to react with the substrate. Bands containing gelatin-degrading MMP-9 appear as clear bands on a dark blue stained background after staining with Coomassie brilliant blue R-250 (Bio-Rad). Both proenzyme and proteolytically activated forms of MMPs were visualized by zymography.

Statistical analysis. Three or more separate experiments were performed. Statistical analysis was performed by paired Student's t-test or ANOVA.

\section{Results}

TM-induced ER stress and PARP cleavage and apoptosis of Caki-2 cells. To investigate the effect of TM on the apoptosis of Caki- 2 cells, cells were treated with TM $(0.01,0.1$ and $5 \mu \mathrm{g} /$ $\mathrm{ml}$ ) in serum-free medium for $24 \mathrm{~h}$. Three established criteria were used to assess apoptosis in our system. Firstly, we examined changes in cell morphologies after TM treatment and observed apoptotic characteristics, such as cell shrinkage and detachment of cells from the plate in a dose-dependent manner (Fig. 1A). Secondly, apoptosis of Caki-2 cells was confirmed using flow cytometric analysis to detect hypo-diploid cell populations with and without TM treatment for $24 \mathrm{~h}$. TM treatment markedly increased the accumulation of sub-G1 phase cells and induced apoptosis in a dose-dependent manner (Fig. 1B and C). Caspase-3 is one of the key executioners of apoptosis. Further, PARP-1 is known to function during DNA 
A

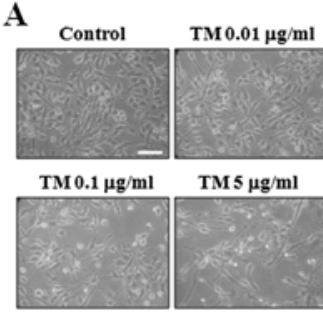

C

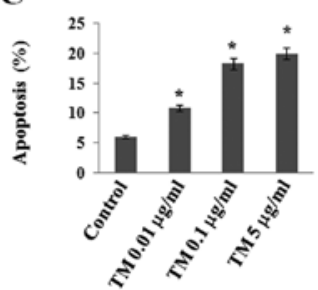

$\mathbf{E}$

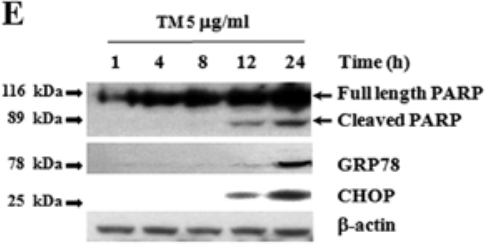

Figure 1. Tunicamycin (TM) induces ER stress along with procaspase-3 and PARP cleavage during apoptosis of Caki- 2 cells. (A) Cells were treated with $\mathrm{TM}$ at different concentrations for $24 \mathrm{~h}$. TM caused morphological changes in cultured cells at $24 \mathrm{~h}$ as determined by phase-contrast microscopy. Scale bar, $50 \mu \mathrm{m}$. (B and C) Flow cytometric and quantitative analyses of apoptotic cells after culture for $24 \mathrm{~h}$. Apoptosis was analyzed as a sub-G1 fraction by FACS. ${ }^{*} \mathrm{P}<0.05$ vs. corresponding value for control. (D) Western blot analysis of PARP and caspase-3 after culture for $24 \mathrm{~h}$. (E) Western blot analysis of ER stress-induced proteins such as GRP78 and CHOP by TM. $\beta$-actin was used as loading control.

repair and to bind DNA strand breaks during apoptosis (26). Thirdly, to determine whether or not caspase-3 activation and PARP cleavage are involved in TM-induced apoptosis, cells were treated with TM $(0.01,0.1$ and $5 \mu \mathrm{g} / \mathrm{ml})$ for $24 \mathrm{~h}$ (Fig. 1D). In western blot analysis with anti-pro-caspase- 3 antibody, high levels of pro-caspase-3 $(32 \mathrm{kDa})$ were detected in cell lysates of the control culture, whereas TM treatment downregulated the expression of pro-caspase- 3 in a dose-dependent manner. Interestingly, pro-caspase-3 protein was not detected at all upon TM treatment at $5 \mu \mathrm{g} / \mathrm{ml}$, which suggests that procaspase- 3 was presumably cleaved into active caspase- 3 (27). Furthermore, we found that the cleavage of PARP increased upon TM treatment in a dose-dependent manner, accompanied by concomitant downregulation of procaspase-3 expression. Further, PARP cleavage was barely detectable in the control culture, which indicates that caspase-3 was involved in TM-induced apoptosis. The ER stress response is involved in the activation of ATF6 and subsequent induction of GRP78 and GADD153 (CHOP), a key component in ER stress-mediated apoptosis (28). Using western blot analysis, we examined whether or not both GRP78 and CHOP are induced during TM-induced apoptosis. For this, cells were treated with $5 \mu \mathrm{g} / \mathrm{ml}$ of TM in serum-free medium for $1,4,8,12$ and $24 \mathrm{~h}$ (Fig. 1E). The protein levels of GRP78 and CHOP were detected after $12 \mathrm{~h}$ of TM treatment, and higher levels of both proteins were clearly detected at $24 \mathrm{~h}$. Simultaneously, we clearly observed PARP cleavage at 12 and $24 \mathrm{~h}$. These results
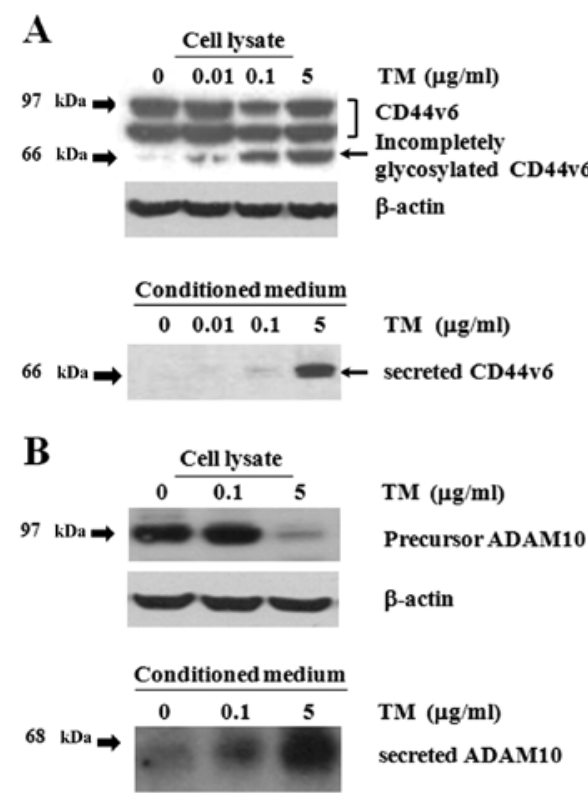

Figure 2. Expression patterns of CD44v6 and ADAM10, and TM-induced secretion of CD44v6 ectodomain and active ADAM10. Western blot analysis was used to examine CD44v6 and ADAM10 expression in cell lysates and conditioned medium after culture for $24 \mathrm{~h}$. (A) Comparable high levels of uncleaved CD44v6 protein (97 and $90 \mathrm{kDa}$ ) were detected in control and TM-treated cells at $24 \mathrm{~h}$. Of note, CD44v6 band (66 kDa; arrow), presumably due to inhibition of N-linked glycosylation, was detected only in TM-treated cells in a dosedependent manner. The secreted form of CD44v6 (66 and $45 \mathrm{kDa}$ ) was highly detected mainly in TM $(5 \mu \mathrm{g} / \mathrm{ml})$-treated cultures at $24 \mathrm{~h}$; these two bands were not detected at all in control cultures. (B) Precursor form of ADAM10 (97 kDa) was detected in control and TM $(0.1 \mu \mathrm{g} / \mathrm{ml})$-treated cell lysates at $24 \mathrm{~h}$, but only a faint band was detected upon $5 \mu \mathrm{g} / \mathrm{ml}$ of TM treatment. Note that high levels of ADAM10 (68 kDa) were present in the collected conditioned medium upon $\mathrm{TM}(5 \mu \mathrm{g} / \mathrm{ml})$ treatment. $\beta$-actin was used as loading control.

indicate that TM-induced ER stress stimulated apoptosis of Caki-2 cells.

TM-induced CD44v6 ectodomain shedding and secretion of ADAM10. Cultured cells interact with a matrix via adhesion molecules for proper function, but TM treatment induced cell detachment from plates in addition to apoptosis (Fig. 1). To measure the levels of CD44v6 as well as its cleaved form, equal amounts of lysate proteins were immunoblotted with a monoclonal anti-CD44v6 antibody. The core protein of $\mathrm{CD} 44 \mathrm{~s}$ is $37 \mathrm{kDa}$ with an apparent molecular mass of $\sim 85 \mathrm{kDa}$ due to glycosylation (22). Extensive post-translational glycosylation of different CD44 variants produces proteins with molecular masses ranging from 80 to $200 \mathrm{kDa}$ (29). Using western blot analysis, high levels of uncleaved CD44v6 (97 and $90 \mathrm{KDa}$ ) were strongly detected in control cell lysates cultured for $24 \mathrm{~h}$ (Fig. 2A). Interestingly, three major bands (97, 90 and $66 \mathrm{kDa}$ ) corresponding to CD44v6 were detected in TM-treated cultures; secreted CD44v6 (66 kDa; arrow) was only detected in TM-treated cells in a dose-dependent manner, presumably due to the inhibition of N-linked glycosylation. Furthermore, to detect shedding of CD44v6 ectodomain, conditioned medium from cells cultured for $24 \mathrm{~h}$ was subjected to western blot analysis (Fig. 2A). As expected, high levels of the secreted form of CD44v6 $(66 \mathrm{kDa})$ were detected in cells treated with TM $(5 \mu \mathrm{g} / \mathrm{ml})$. 
A
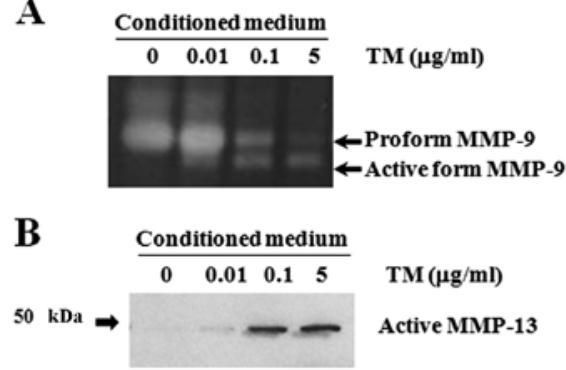

Figure 3. Induction of MMP-9 activation and expression of active MMP-13 by TM. (A) Gelatin zymography using conditioned medium was performed to detect MMP-9. In control and TM $(0.01 \mu \mathrm{g} / \mathrm{ml})$-treated cultures at $24 \mathrm{~h}$, the pro-form of MMP-9 $(92 \mathrm{kDa})$ was highly detected in conditioned medium. Note that TM $(0.1$ and $5 \mu \mathrm{g} / \mathrm{ml})$ treatment induced the active form of MMP-9. (B) Western blot analysis showed that TM $(0.1$ and $5 \mu \mathrm{g} / \mathrm{ml})$ treatment strongly induced secretion of the active form of MMP-13 (48 kDa). $\beta$-actin was used as loading control.

In contrast, secretion of CD44v6 was not detected at all in control cultures. Figs. 1 and 2 together indicate that cleavage of CD44v6 ectodomain is regulated during TM-induced apoptosis of Caki-2 cells.

ADAM10 is expressed as an inactive pro-form $(97 \mathrm{kDa})$, which is later processed to a shorter, active form (68 kDa) (22). We next examined the presence of active and secreted forms of ADAM10 in TM-treated cultures (Fig. 2B). Using western blot analysis, only the single $97-\mathrm{kDa}$ protein was detected in cell lysates of control and TM $(0.1 \mu \mathrm{g} / \mathrm{ml})$-treated cultures at $24 \mathrm{~h}$. In contrast, the $97-\mathrm{kDa}$ protein was barely detectable in lysates of TM $(5 \mu \mathrm{g} / \mathrm{ml})$-treated cells. Although ADAM10 is a membrane-anchored glycoprotein, it is present in the pericellular ECM of the developing corneal stroma, and the secreted form is detected in cultured cells (21). Further, we found high levels of the $68-\mathrm{kDa}$ band present in the collected conditioned medium in TM $(5 \mu \mathrm{g} / \mathrm{ml})$-treated cells, showing that the active form of ADAM10 was secreted. These data suggest that CD44v6 ectodomain cleavage was mediated at least in part by active ADAM10 during TM-induced apoptosis.

TM-stimulated activation of MMP-9 and expression of active MMP-13. CD44 serves as a docking molecule to retain MMP-9 activity at the cell surface (30), and it leads to activation of pro-MMP-9 (31) and MMP-9 during secretion (32). MMP-9induced CD44 cleavage can be inhibited by an MMP-9 inhibitor (33). To determine whether or not a causal relationship exists between MMP-9 activity and CD44v6 cleavage, gelatin zymography was performed to detect MMP-9 protein (Fig. 3A). In control culture, the pro-form of MMP-9 (92 kDa) was detected at high levels in conditioned medium, whereas the active form $(82 \mathrm{kDa})$ was not detected at all. In contrast, both the pro- and active forms of MMP-9 were detected upon $0.1 \mu \mathrm{g} / \mathrm{ml}$ of TM treatment. Interestingly, pro-MMP-9 activity dramatically declined and became undetectable, whereas the active form of MMP-9 was observed upon $5 \mu \mathrm{g} / \mathrm{ml}$ of TM treatment. These data indicate that MMP-9 underwent proteolysis consistent with its activation during apoptosis in response to TM treatment. Given the evidence that MMP-13 is able to activate pro-MMP-9 to MMP-9 (25), we examined the level of active MMP-13
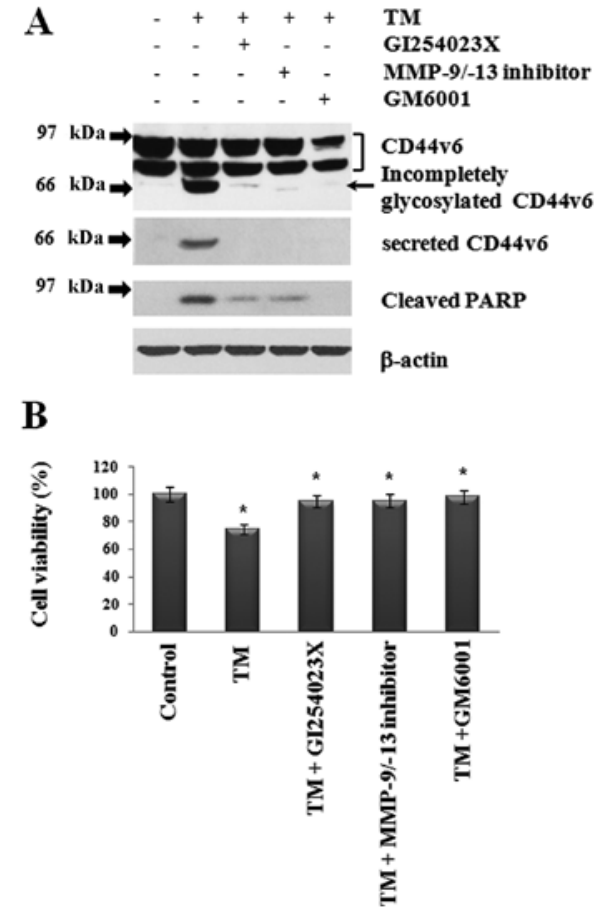

Figure 4. Inhibition of TM-induced PARP cleavage and apoptosis by several MMP inhibitors. Cells were cultured with $5 \mu \mathrm{g} / \mathrm{ml}$ of TM in the presence of $20 \mu \mathrm{M}$ GM6001 (a broad spectrum synthetic MMP inhibitor), $500 \mu \mathrm{M}$ MMP-9/MMP-13 inhibitor and $10 \mu \mathrm{M}$ GI254023X (specific ADAM10 inhibitor) for $24 \mathrm{~h}$. (A) Western blot analysis of PARP cleavage using cell lysates. Note that the level of TM-induced PARP cleavage dramatically decreased upon treatment with ADAM10 or MMP-9/-13 inhibitors and could not be detected in the GM6001-treated sample. (B) Analysis of cell viability by MTT assay. Note that TM-induced apoptosis was restored by treatment with all MMP inhibitors. $\beta$-actin was used as loading control.

protein during TM-induced apoptosis of Caki-2 cells using conditioned medium by western blot analysis (Fig. 3B). High levels of proteolytically active MMP-13 protein $(48 \mathrm{kDa})$ were strongly detected in TM $(0.1$ and $5 \mu \mathrm{g} / \mathrm{ml})$-treated cells at $24 \mathrm{~h}$. In contrast, no MMP-13 protein was detected in control cells, suggesting a possible role for MMP-13 in MMP-9 activation.

Inhibition of TM-induced PARP cleavage and apoptosis by treatment with MMP inhibitors. TM-induced PARP cleavage and MMP activation occurred in a dose-dependent manner during apoptosis (Figs. 1-3). Therefore, we further examined whether or not inhibition of MMP-9, MMP-13 and ADAM10 in TM-treated cells decreases both CD44v6 ectodomain and PARP cleavage while increasing cell viability (Fig. 4). As expected, TM-induced cleavage of CD44v6 and PARP at $24 \mathrm{~h}$ was greatly reduced by treatment with MMP-9/-13 inhibitor, ADAM10-specific inhibitor (GI254023X) and GM6001, which is a broad spectrum MMP inhibitor (Fig. 4A). Surprisingly, incomplete glycosylation of CD44v6 induced by TM was also abrogated by treatment with all MMP inhibitors. Furthermore, we performed MTT assay to check cell viability (Fig. 4B). As expected, TM-induced apoptosis was reduced by all MMP inhibitors. These data indicate that TM-induced apoptosis was mediated in part by MMP-9, MMP-13 and ADAM10 functions. 


\section{Discussion}

In the present study, we examined the connection between MMP expression/activity and apoptosis, and we found that MMP-9, MMP-13 and ADAM10 are intimately involved in TM-induced cleavage of CD44v6 ectodomain and apoptosis of Caki-2 cells. We further provide direct evidence that TM induces MMP-9 activation and expression of active MMP-13 and ADAM10, and that inhibition of ADAM10 or MMP-9/ MMP-13 activity increases Caki-2 cell viability.

Induction of cleavage of CD44v6 ectodomain during TM-induced cell apoptosis. CD44 functionally interacts with growth factors deposited in the ECM (7) and growth factor receptors, which trigger activation of signal transduction cascades $(13,34)$, thereby regulating cell proliferation. On the cytoplasmic side, the interaction between CD44 and NF2 proteins can be pivotal in determining whether cells proliferate or undergo apoptosis (35). Normal cells expressing CD44s are susceptible to cleavage of PARP and apoptosis (36). In contrast, CD44v6 coordinates activation of anti-apoptotic molecules and mediates apoptosis resistance via the PI3K/Akt and ras-raf-MAPK pathways (13). In addition, cells expressing high levels of CD44v6 are resistant to PARP cleavage as well as Fas-mediated apoptosis depending on the cell type (14). Therefore, we can assume that high levels of CD44v6 are constitutively expressed in Caki-2 cells for the purpose of apoptosis resistance (Fig. 2). Treatment with TM either slightly reduced or did not affect cell surface CD44 expression, but it has been shown to inhibit hyaloranate binding by CD 44v and destroy clustering of CD44v6 in carcinoma cells $(37,38)$. Likewise, high levels of uncleaved CD44v6 (97 and $90 \mathrm{kDa}$ ) were detected similarly in both control and TM-treated cultures (Fig. 2A). On the other hand, when cells were treated with $5 \mu \mathrm{g} / \mathrm{ml}$ of TM, presumably a de-glycosylated CD44v6 (66-KDa) band was strongly detected in cell lysates. However, a CD44v6 (66-kDa) band was not detected at all in control cultures. Soluble CD44 and CD44v6 have been identified in cultured supernatants in human prostate tumor cell lines (39). In this study, high levels of soluble CD44v6 ectodomain $(66 \mathrm{kDa})$ were detected only in TM $(5 \mu \mathrm{g} / \mathrm{ml})$-treated conditioned medium (Fig. 2A), suggesting that this band represents the cleaved form of whole CD44v6 (97 or $90 \mathrm{kDa}$ ) and not the incompletely glycosylated form of CD44v6 (66 kDa) detected in cell lysates. Soluble CD44 can compete with cancer cell membrane CD44 for matrix-binding sites and exert anticancer effects, including decreased tumorigenicity and increased apoptosis (40). Although we demonstrated changes in CD44v6 glycosylation upon treatment with TM, we do not yet know whether or not de-glycosylation of other molecules on the cell surface influences CD44v6 function. Collectively, our present data indicate that incomplete glycosylation of CD44v6 as well as ectodomain shedding by TM may play roles in the apoptosis of Caki-2 cells.

Sequential proteolytic cleavages in the ectodomain and intramembranous domain of CD44 play critical roles in various disease pathologies (41). ADAM10 serves as the constitutive functional sheddase of CD44 in several melanoma cell lines, and soluble CD44 can abolish cell proliferation (20). In addition, silencing of ADAM10, but not MMP-14 (MT1-MMP), stimulates cell proliferation in a CD44-dependent manner (20). Note that the secreted form of active ADAM10 (68 kDa) was highly abundant upon $5 \mu \mathrm{g} / \mathrm{ml}$ of TM treatment during cleavage of CD44v6 ectodomain and PARP at $24 \mathrm{~h}$ (Fig. 2B). Furthermore, inhibition of ADAM10 activity by GI254023X treatment reduced TM-induced PARP cleavage and increased the viability of Caki-2 cells (Fig. 4). Taken together, our study suggests that the secreted form of active ADAM10 may be involved in the cleavage of CD44v6 ectodomain, which modulates apoptosis of Caki-2 cells.

Induction of active MMP-13 and activation of MMP-9 during TM-induced apoptosis. Pro-MMP-9 is activated by osteoarthritic chondrocytes via the MMP-13 cascade (24). In addition, MMP-13 can initiate bone resorption and activate pro-MMP-9 in vitro, and MMP-13 inhibitor prevents MMP-9 activation (25). Pro-MMP-9 was converted into active MMP-9 by TM treatment in a dose-dependent manner (Fig. 3A). Importantly, the temporal expression pattern of active MMP-13 protein was correlated with MMP-9 activation (Fig. 3B). Inhibition of caspase suppresses induction of MMP-9 expression (42). Further, as PARP-1 is involved in the transcriptional activation of MMP-9, induction of apoptosis through caspase activation and PARP-1 cleavage results in re-repression of MMP-9 promoter activity (42). In our study, inhibition of both MMP-9 and MMP-13 reduced TM-induced PARP cleavage, but increased viability of Caki-2 cells (Fig. 4). Taken together (Figs. 3 and 4), the active forms of both MMP-9 and MMP-13 exhibit similar temporal expression patterns, which may be correlated with TM-induced apoptosis.

CD44 retains MMP-9 activity at the cell surface (30), and surface expression of CD44 along with activation of MMP-9 on the cell surface are interdependent (9). In addition, soluble CD44 mediates secretion of MMP-9 (32). The CD44v6-matrix interaction stimulates MMP-9 production and promotes MMP-9 binding to CD44 (43). MMP-9 acts as a processing enzyme for CD44 cleavage, which is inhibited by both transcriptional knockdown of MMP-9 and MMP-9 specific inhibitor (33). Collectively, our findings of TM-mediated active MMP-13 protein induction and MMP-9 activation in combination with caspase-3 activation and PARP cleavage (Figs. 1, 3, and 4) suggest that TM may participate in the regulation of CD44v6 cleavage via MMP-9 and MMP-13 during the apoptosis of Caki- 2 cells.

In summary, these findings collectively support our hypothesis that apoptosis of Caki-2 cells in vitro is mediated by the temporal regulation of multiple active MMPs (e.g., MMP-9, MMP-13 and ADAM10). We further show that TM-induced CD44v6 ectodomain cleavage and apoptosis occurs through an MMP-dependent mechanism. Further studies will be necessary to understand the precise molecular mechanism of these effects.

\section{Acknowledgements}

This study was supported by the Basic Science Research Program of the National Research Foundation of Korea (NRF), which is funded by the Ministry of Education, Science and Technology (2011-0004933). This work was also supported by a Korean Research Foundation Grant (KRF-2006-521-C00148 and KRF-2009-0075108). 


\section{References}

1. Dolai S, Pal S, Yadav RK and Adak S: Endoplasmic reticulum stress-induced apoptosis in Leishmania through $\mathrm{Ca}^{2+}$-dependent and caspase-independent mechanism. J Biol Chem 286: 13638-13646, 2011.

2. Elbein AD: Inhibitors of the biosynthesis and processing of N-linked oligosaccharide chains. Annu Rev Biochem 56: 497-534, 1987.

3. Delom F, Fessart D and Chevet E: Regulation of calnexin sub-cellular localization modulates endoplasmic reticulum stressinduced apoptosis in MCF-7 cells. Apoptosis 12: 293-305, 2007.

4. Dricu A, Carlberg M, Wang M and Larsson O: Inhibition of $\mathrm{N}$-linked glycosylation using tunicamycin causes cell death in malignant cells: role of down-regulation of the insulin-like growth factor 1 receptor in induction of apoptosis. Cancer Res 57: 543-548, 1997.

5. Hitomi J, Katayama T, Taniguchi M, Honda A, Imaizumi K and Tohyama M: Apoptosis induced by endoplasmic reticulum stress depends on activation of caspase-3 via caspase-12. Neurosci Lett 357: 127-130, 2004.

6. Nakagawa T, Zhu H, Morishima N, et al: Caspase-12 mediates endoplasmic-reticulum-specific apoptosis and cytotoxicity by amyloid- $\beta$. Nature 403: 98-103, 2000.

7. Yu Q and Stamenkovic I: Localization of matrix metalloproteinase 9 to the cell surface provides a mechanism for CD44-mediated tumor invasion. Genes Dev 13: 35-48, 1999.

8. Arch R, Wirth K, Hofmann M, et al: Participation in normal immune responses of a metastasis-inducing splice variant of CD44. Science 257: 682-685, 1992.

9. Desai B, Ma T, Zhu J and Chellaiah MA: Characterization of the expression of variant and standard CD44 in prostate cancer cells: identification of the possible molecular mech+anism of CD44/ MMP9 complex formation on the cell surface. J Cell Biochem 108: 272-284, 2009.

10. Naor D, Sionov RV and Ish-Shalom D: CD44: structure, function, and association with the malignant process. Adv Cancer Res 71: 241-319, 1997.

11. Yamane N, Tsujitani S, Makino M, Maeta M and Kaibara N: Soluble CD44 variant 6 as a prognostic indicator in patients with colorectal cancer. Oncology 56: 232-238, 1999.

12. Wielenga VJ, Heider KH, Offerhaus GJ, et al: Expression of CD44 variant proteins in human colorectal cancer is related to tumor progression. Cancer Res 53: 4754-4756, 1993.

13. Jung T, Gross W and Zoller M: CD44v6 coordinates tumor matrix-triggered motility and apoptosis resistance. J Biol Chem 286: 15862-15874, 2011.

14. Mielgo A, van Driel M, Bloem A, Landmann L and Gunthert U: A novel antiapoptotic mechanism based on interference of Fas signaling by CD44 variant isoforms. Cell Death Differ 13: 465-477, 2006

15. Fini ME and Girard MT: The pattern of metalloproteinase expression by corneal fibroblasts is altered by passage in cell culture. J Cell Sci 97: 373-383, 1990.

16. Girard MT, Matsubara M, Kublin C, Tessier MJ, Cintron C and Fini ME: Stromal fibroblasts synthesize collagenase and stromelysin during long-term tissue remodeling. J Cell Sci 104: 1001-1011, 1993.

17. Sternlicht MD and Werb Z: How matrix metalloproteinases regulate cell behavior. Annu Rev Cell Dev Biol 17: 463-516, 2001.

18. Kessenbrock K, Plaks V and Werb Z: Matrix metalloproteinases: regulators of the tumor microenvironment. Cell 141: 52-67, 2010.

19. Smith KM, Gaultier A, Cousin H, Alfandari D, White JM and DeSimone DW: The cysteine-rich domain regulates ADAM protease function in vivo. J Cell Biol 159: 893-902, 2002.

20. Anderegg U, Eichenberg T, Parthaune T, et al: ADAM10 is the constitutive functional sheddase of CD44 in human melanoma cells. J Invest Dermatol 129: 1471-1482, 2009.

21. Huh MI, Lee YM, Seo SK, et al: Roles of MMP/TIMP in regulating matrix swelling and cell migration during chick corneal development. J Cell Biochem 101: 1222-1237, 2007.

22. Nagano O, Murakami D, Hartmann D, et al: Cell-matrix interaction via CD44 is independently regulated by different metalloproteinases activated in response to extracellular $\mathrm{Ca}(2+)$ influx and PKC activation. J Cell Biol 165: 893-902, 2004.
23. Pacheco-Rodriguez G, Steagall WK, Crooks DM, et al: TSC2 loss in lymphangioleiomyomatosis cells correlated with expression of CD44v6, a molecular determinant of metastasis. Cancer Res 67: 10573-10581, 2007.

24. Dreier R, Grassel S, Fuchs S, Schaumburger J and Bruckner P: Pro-MMP-9 is a specific macrophage product and is activated by osteoarthritic chondrocytes via MMP-3 or a MT1-MMP/ MMP-13 cascade. Exp Cell Res 297: 303-312, 2004.

25. Hernandez Rios M, Sorsa T, Obregon F, et al: Proteolytic roles of matrix metalloproteinase (MMP)-13 during progression of chronic periodontitis: initial evidence for MMP-13/MMP-9 activation cascade. J Clin Periodontol 36: 1011-1017, 2009.

26. Lazebnik YA, Kaufmann SH, Desnoyers S, Poirier GG and Earnshaw WC: Cleavage of poly(ADP-ribose) polymerase by a proteinase with properties like ICE. Nature 371: 346-347, 1994.

27. Ferreira KS, Kreutz C, Macnelly S, et al: Caspase-3 feeds back on caspase-8, Bid and XIAP in type I Fas signaling in primary mouse hepatocytes. Apoptosis 17: 503-515, 2012.

28. Oyadomari S and Mori M: Roles of CHOP/GADD153 in endoplasmic reticulum stress. Cell Death Differ 11: 381-389, 2004

29. Ponta H, Sleeman J, Dall P, Moll J, Sherman L and Herrlich P. CD44 isoforms in metastatic cancer. Invasion Metastasis 14: 82-86, 1994.

30. Spessotto P, Rossi FM, Degan M, et al: Hyaluronan-CD44 interaction hampers migration of osteoclast-like cells by downregulating MMP-9. J Cell Biol 158: 1133-1144, 2002.

31. Samanna V, Ma T, Mak TW, Rogers M and Chellaiah MA: Actin polymerization modulates CD44 surface expression, MMP-9 activation, and osteoclast function. J Cell Physiol 213: 710-720, 2007.

32. Seipel D, Oliveira BC, Resende TL, et al: Toxoplasma gondii infection positively modulates the macrophages migratory molecular complex by increasing matrix metalloproteinases, CD44 and alpha v beta 3 integrin. Vet Parasitol 169: 312-319, 2010.

33. Chetty C, Vanamala SK, Gondi CS, Dinh DH, Gujrati M and Rao JS: MMP-9 induces CD44 cleavage and CD44 mediated cell migration in glioblastoma xenograft cells. Cell Signal 24: 549-559, 2012.

34. Ponta H, Sherman L and Herrlich PA: CD44: from adhesion molecules to signalling regulators. Nat Rev Mol Cell Biol 4: 33-45, 2003.

35. Morrison H, Sherman LS, Legg J, et al: The NF2 tumor suppressor gene product, merlin, mediates contact inhibition of growth through interactions with CD44. Genes Dev 15: 968-980, 2001.

36. Wittig BM, Johansson B, Zoller M, Schwarzler C and Gunthert U: Abrogation of experimental colitis correlates with increased apoptosis in mice deficient for CD44 variant exon 7 (CD44v7). J Exp Med 191: 2053-2064, 2000.

37. Lesley J, English N, Perschl A, Gregoroff J and Hyman R: Variant cell lines selected for alterations in the function of the hyaluronan receptor CD44 show differences in glycosylation. J Exp Med 182: 431-437, 1995.

38. Sleeman J, Rudy W, Hofmann M, Moll J, Herrlich P and Ponta H: Regulated clustering of variant CD44 proteins increases their hyaluronate binding capacity. J Cell Biol 135: 1139-1150, 1996.

39. Stevens JW, Palechek PL, Griebling TL, Midura RJ, Rokhlin OW and Cohen MB: Expression of CD44 isoforms in human prostate tumor cell lines. Prostate 28: 153-161, 1996.

40. Yu Q, Toole BP and Stamenkovic I: Induction of apoptosis of metastatic mammary carcinoma cells in vivo by disruption of tumor cell surface CD44 function. J Exp Med 186: 1985-1996, 1997.

41. Isacke CM and Yarwood $\mathrm{H}$ : The hyaluronan receptor, CD44. Int J Biochem Cell Biol 34: 718-721, 2002.

42. Kobayashi T: Suppression of matrix metalloproteinase- 9 expression in undifferentiated, non-apoptotic keratinocytes is abrogated by the cleavage of poly(ADP-ribose) polymerase-1. Apoptosis 16: 1205-1216, 2011.

43. Yu Q and Stamenkovic I: Transforming growth factor-beta facilitates breast carcinoma metastasis by promoting tumor cell survival. Clin Exp Metastasis 21: 235-242, 2004. 